\title{
Modulation of defect modes intensity by controlled light scattering in photonic crystal with liquid crystal domain structure
}

\author{
V.A. Gunyakov ${ }^{\mathrm{a}, *}$, M.N. Krakhalev ${ }^{\mathrm{a}, \mathrm{b}}$, V.Ya. Zyryanov ${ }^{\text {a }}$, V.F. Shabanov ${ }^{\text {a }}$, V.A. Loiko ${ }^{\mathrm{c}}$ \\ ${ }^{a}$ Kirensky Institute of Physics, Krasnoyarsk Scientific Center, Siberian Branch of Russian Academy \\ of Sciences, Krasnoyrsk 660036, Russia \\ ${ }^{\mathrm{b}}$ Siberian Federal University, Krasnoyarsk 660041, Russia \\ c Stepanov Institute of Physics, National Academy of Sciences of Belarus, Minsk 220072, Belarus \\ *E-mail address: gun@iph.krasn.ru (V.A. Gunyakov)
}

We propose a technique for modulating the intensity of defect modes in a multilayer photonic structure containing a nematic liquid crystal with the spatially extended director field as a defect layer. The technique uses the domain grid regime of electrohydrodynamic convection in nematics, which leads to the occurrence of a polarization-sensitive scattering mode in the optical response. The control parameters are the variation both of the voltage and the angle between the initial director orientation and the light polarization.

Keywords: Photonic crystal, Liquid crystal, Spatial extended system, Electrohydrodynamic instability, Light scattering

\section{Introduction}

Photonic-crystal (PC) structures attract much attention as new optical materials whose permittivity periodically changes in one, two, of three dimensions with the spatial scale comparable with the light wavelength $[1,2]$. An important property of these structures is the presence of photonic band gaps (PBGs) with the low density of photonic states and low transmittance. The band gaps exhibit extraordinary dispersion characteristics, which allow implementing some regimes of propagation of light waves in the PC structures that are interesting for both fundamental research and application [1-3]. One-dimensional (1D) photonic crystals are multilayer periodic structures consisting of alternating layers of dielectric materials with different refractive indices [4]. In contrast to 3D-PCs, they do not have a complete PBG; however, they are multifunctional and simple to fabricate. Such structures are widely used as interference filters, antireflection coatings, mirrors with high reflectance in a wide frequency range, etc. In band gaps of the photonic crystals with a lattice defect, i.e., broken periodicity, transmission bands occur that are called the defect or localized modes. Combining PCs and liquid crystals (LCs) as a defect layer, one can obtain photonic structures with controllable spectral characteristics [3, 4]. High sensitivity of LCs to external factors (temperature and electric or magnetic fields) in combination with high 
birefringence, transparency in the visible and near-infrared ranges, and optical nonlinearity make it possible to control the spectral position and transmittance of defect modes in these structures. The multilayer PC structures with the tunable spectrum have been well-studied [5-7], but there is a lack of works devoted to the methods for controlling the defect mode amplitude and related intensity modulation. High-contrast modulation of defect modes in a PC/LC structure controlled by electric or magnetic field was experimentally demonstrated in [8-10]. The proposed technique is based on the field-induced matching of the tunable $e$-modes, corresponding to extraordinary light waves, with fixed $o$-modes, corresponding to ordinary light waves. The matching can lead to both interference amplification of their intensity and mutual quenching of the modes of two types. These interference effects cannot be implemented without additional polarization elements. On the other hand, of interest are recent studies on 1D-PCs characterized by scattering of light waves on optical inhomogeneities arisen [11] or inserted [12] in defects of the periodic structure. The authors of [11] developed the electric-field-controlled photonic device with no polarization elements on the basis of a 1D-PC infiltrated with a double-frequency cholesteric LC as a defect layer. The device allows using different frequency-modulated voltage pulses for controlling defect modes and switching the stable states. The authors of [12] theoretically and experimentally investigated transmission of light through a Fabry-Perot microcavity consisting of distributed mirrors and containing a dielectric cylindrical rod as a defect. It was shown that the light transmitted through this system is scattered at different resonance angles and undergoes the angular resonance transformation. As controlled defects in PCs, the so-called spatially extended systems can be used, including nematic LCs characterized by the electroconvective instabilities. It is caused by the spatial inhomogeneity of optical anisotropy of LC in the originally homogeneous nematic liquid crystal caused by the applied field over the threshold value [13]. The approach to describe scattering processes in the domains generated by the electric field is similar to the approach for description of light scattering by a LC droplet in polymer $[14,15]$. The difference is that formed domains change their dimensions and they are in anisotropic "matrix" of liquid crystal.

The hierarchy of convective structures easily switched by electric field and the dependence of the optical response of spatially extended nematics (SEN) on light polarization make it possible to control the amplitude of modes in the PC spectrum.

In this study, we propose a new way of modulation of the mode intensity based on controlled light scattering in a multilayer PC/SEN structure. The electrohydrodynamic convection, which manifests itself as a domain grid pattern (GP) in nematic, is used. In addition the continuous variation in the transmittance of defect modes in the spectrum of the photonic structure is initiated by changing the angle $\alpha$ between orientation of the nematic director $\mathbf{n}$ on the substrates and light polarization via rotation of a polarizing element. 


\section{Photonic structure}

A scheme of the photonic structure used for studying the effect of different electroconvective instabilities on its spectral characteristics is presented in Fig. 1a. A sample consists of two dielectric mirrors with a gap of $11.7 \mu \mathrm{m}$ filled with the 4-metoxybenzylidene-4'butylaniline (MBBA) nematic LC, which has the negative permittivity anisotropy $\left(\varepsilon_{a}<0\right)$ and positive conductivity anisotropy $\left(\sigma_{a}>0\right)$. The clearing temperature of nematic is $T_{c}=45^{\circ} \mathrm{C}$ and the refractive indices are $n_{\mathrm{e}}=1.765$ and $n_{\mathrm{o}}=1.552\left(t=23^{\circ} \mathrm{C}, \lambda=589 \mathrm{~nm}\right)$ for the light polarized parallel and perpendicular to the director, respectively. The multilayer coating of the mirrors consists of six 55-nm-thick zirconium dioxide $\left(\mathrm{ZrO}_{2}\right)$ layers with a refractive index of 2.04 and five 102-nm-thick silicon dioxide $\left(\mathrm{SiO}_{2}\right)$ layers with a refractive index of 1.45 alternately deposited onto the surface of quartz substrates preliminary coated with a thin $(\sim 150 \mathrm{~nm})$ ITO layer, which serves as an electrode for applying electric field $\mathbf{E}$ normally to the sample plane. The planar orientation $\mathbf{n}$ of the LC director was specified by an unidirectionally rubbed polymer coating. The orientation was controlled on a polarization microscope by extinction of the field of view in crossed polarizers. Hereinafter, the light wave polarizations parallel and perpendicular to the rubbing direction in all the microscopic, spectral, and electrooptical measurements are denoted by indices $\|$ and $\perp$, respectively. The applied ac field with a frequency of $80 \mathrm{~Hz}$ below a certain threshold value cannot modify the optical response of the photonic structure with the uniformly ordered nematic LC (Fig. 1b). Above the threshold, the nematic structure is periodically disturbed due to the electrohydrodynamic convection, which causes strong light scattering (Fig. 1c).

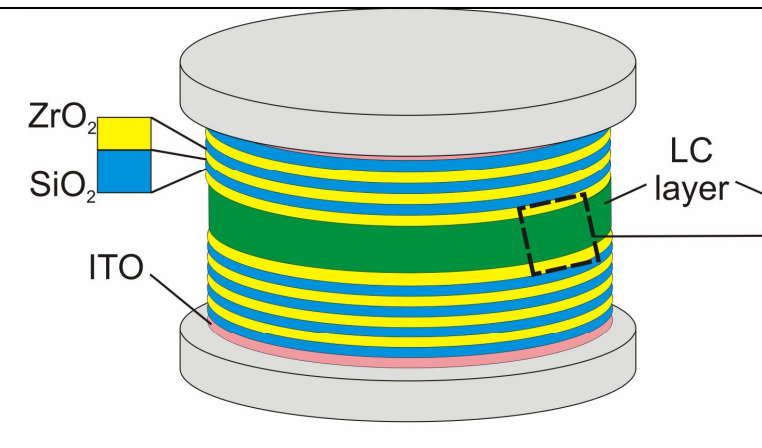

(a)

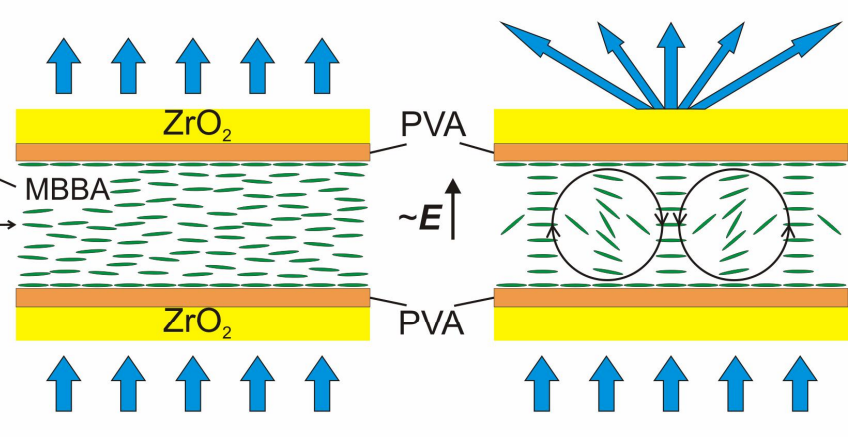

(b)

(c)

Fig. 1. Scheme of an electrically controlled PC/SEN cell with ITO coating under $\mathrm{ZrO}_{2} / \mathrm{SiO}_{2}$ multilayers (a). The LC layer is nematic MBBA with $11.7 \mu \mathrm{m}$ thickness. Orientation of the LC director when applied ac field is less than the threshold value (b). Orientation of the LC director when applied ac field is more than the threshold value (c). In case (b) there is a planar orientation and scattering is absent, in case (c) electroconvective instability is implemented which causes light scattering. 


\section{Experiment results and discussion}

Microphotographs of the LC textures in the photonic structure obtained using a polarizing microscope (Figs. 2a-2e) show the transformation of different electroconvection patterns in the nematic with increasing ac voltage. The upper and lower rows of the textures correspond to the parallel $(\|)$ and perpendicular $(\perp)$ polarizations of the light wave, respectively. For $\|$-component, the structure remains transparent up to the threshold voltage $U_{\mathrm{c}}=6.2 \mathrm{~V}$ (Fig. 2a) due to the uniform alignment of nematic molecules in the defect layer. The primary electroconvective pattern, the socalled Williams domains (WD), occurs at the threshold field (Fig. 2b) [16]. Convection leads to the spatial periodicity of the LC refractive index and thus causes spatial modulation of the light field phase on an output mirror. Then, as the applied ac voltage is increased, one observes secondary instability such as oblique rolls (OR) instability (Fig. 2c) [17], which are brought to the domain grid pattern (Fig. 2d) [18]. The grid pattern is the last ordered state in the nematic liquid flow, which is followed by the chaotic dynamic scattering mode (DSM) accompanied by the strong light scattering (Fig. 2e). Note that $\perp$-component remains transparent up to a voltage of $6.5 \mathrm{~V}$ (Fig. $2 \mathrm{c}$ ) above which the textures of this polarization repeat the convective patterns characteristic of $\|$-component, but with the much weaker contrast.

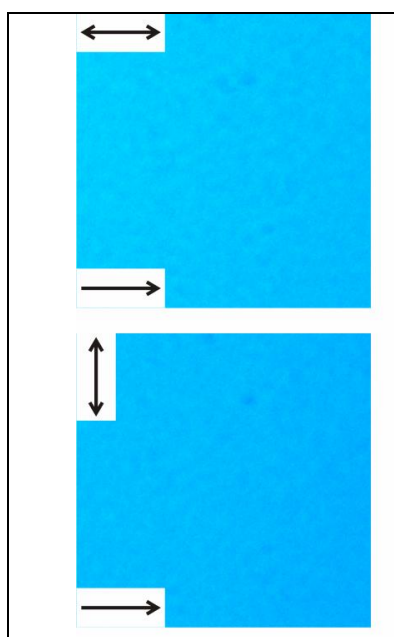

(a)
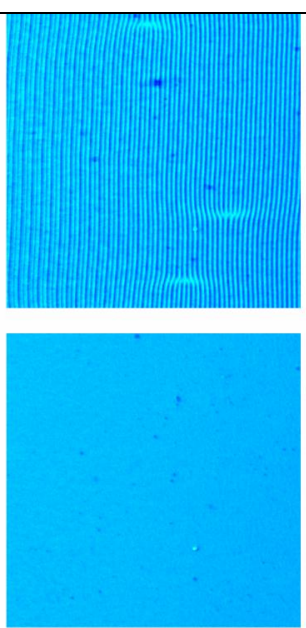

(b)
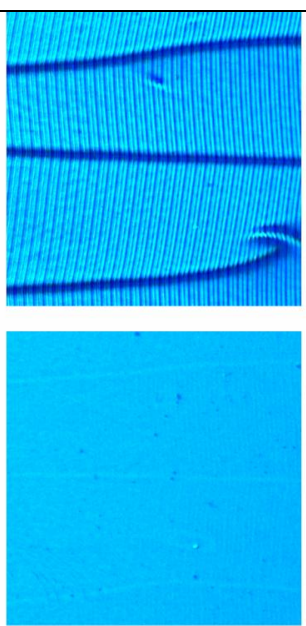

(c)

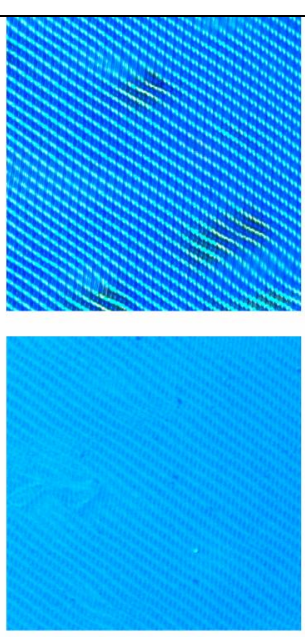

(d)

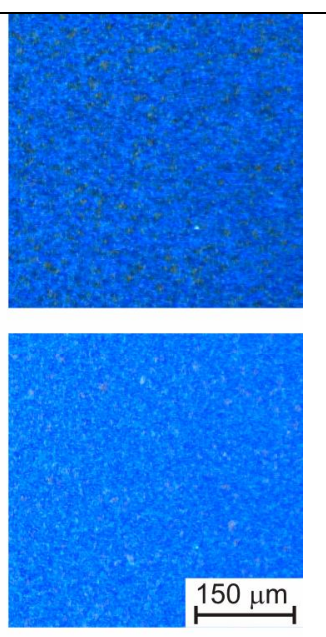

(e)

Fig. 2. Microphotographs of LC textures in photonic structure at applied ac voltage: $U=6.0 \mathrm{~V}$ (a), $U=6.2 \mathrm{~V}(\mathrm{~b}), U=6.7 \mathrm{~V}(\mathrm{c}), U=7.7 \mathrm{~V}(\mathrm{~d}), U=12.0 \mathrm{~V}$ (e). Arrows indicate the direction of light polarization $(\leftrightarrow)$ and the LC director $\mathbf{n}(\rightarrow)$, respectively. 
The polarized components of the transmission spectrum $T_{\|, \perp}(\lambda)$ and the field dependences of the spectra $T_{\|, \perp}(U)$ under the conditions of the discussed convection processes were studied using a Shimadzu UV-3600 spectrometer. As a polarizing element, we used a Glan prism oriented along (\|component) or perpendicular ( $\perp$-component) to the rubbing direction by its principle plane. An aperture $4 \mathrm{~mm}$ in diameter was placed behind the sample. Spectra were detected at a fixed temperature of $25^{\circ} \mathrm{C}$; the sample thermal stabilization accuracy was no worse than $\pm 0.2^{\circ} \mathrm{C}$. The investigated PC structure forms a PBG in the transmission spectrum at wavelengths of $420-610 \mathrm{~nm}$ with a set of localized modes whose position is determined by the parameters of mirrors and defect layer and by the direction of polarization of the probing radiation. The transmission spectrum of the PC with the planar MBBA layer obtained at an applied ac voltage below threshold voltage $U_{\mathrm{c}}$ forms two sets of defect modes corresponding to components $T_{\|, \perp}$ (Fig. 3).

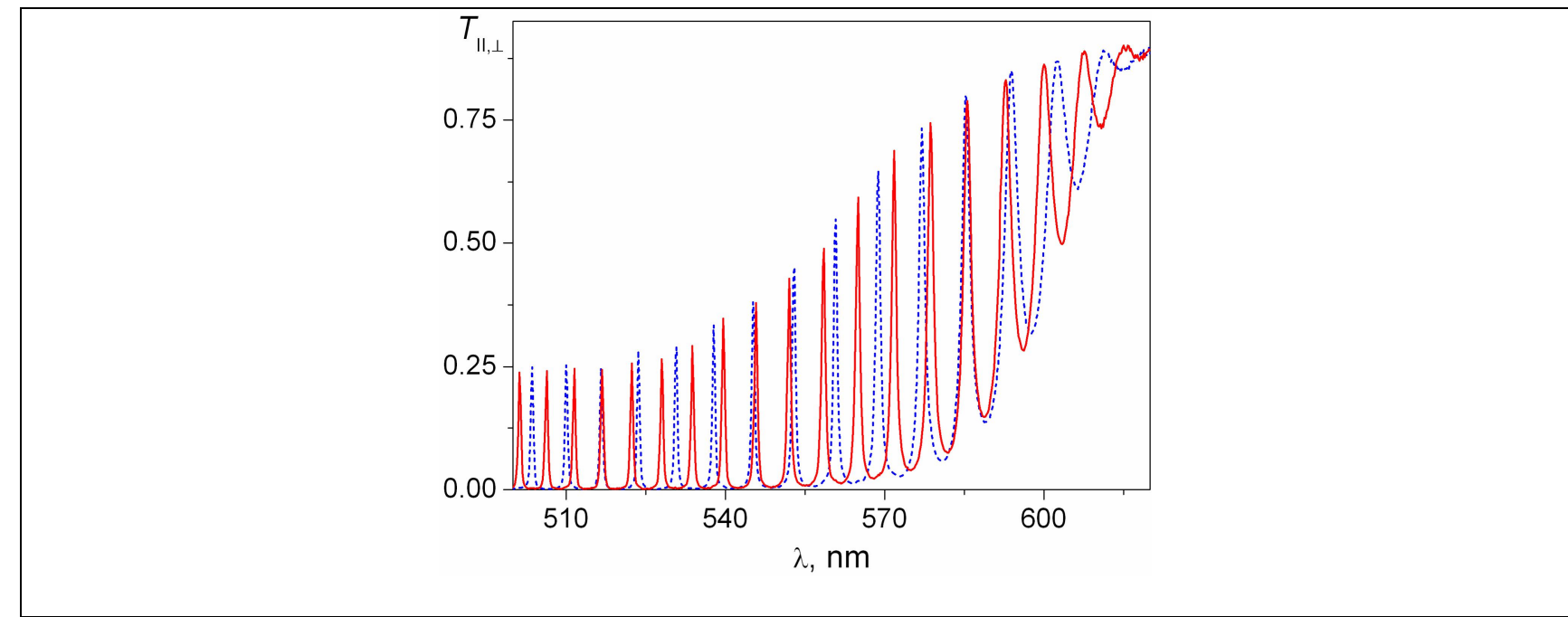

Fig. 3. Polarized components $T_{\|}$(solid line) and $T_{\perp}$ (dashed line) of the PC/LC transmission spectrum measured at temperature $t=25^{\circ} \mathrm{C}$. Electric field is switched off.

Transformation of the spectra above threshold voltage $U_{\mathrm{c}}$ in the wavelength range 500-520 $\mathrm{nm}$ at the PBG center, which is most sensitive to the radiation loss in real PC structures [7] is shown in Fig.4. As one can see the field dynamics of damping of $\|$ - and $\perp$-modes is essentially different. In addition, in contrast to the transverse modes, the parallel modes noticeably shift to the short-wavelength range from their initial position: at an average mode half-width of $0.5 \mathrm{~nm}$, the half a volt voltage increment leads to the shift by $1 \mathrm{~nm}$. This is due to an increase in the depth of modulation of director orientation $\mathbf{n}$ in the LC bulk and an decrease in its effective refractive index $n_{\text {eff }}(\theta)$ for $\|$-component, where $\theta$ is the angle between the electric field vector and local director [19]. In the discussed case, the well-known relation between the spectral position of the mode and the refractive index of the medium in a Fabry-Perot cavity is written as 


$$
\lambda_{e}=\left\{\begin{array}{ll}
2 L n_{e} / m=\text { const }, & U<U_{c} \\
2 L n_{e f f} / m, & U \geq U_{c}
\end{array},\right.
$$

where $L$ is the cavity thickness and $m$ is the defect mode number.

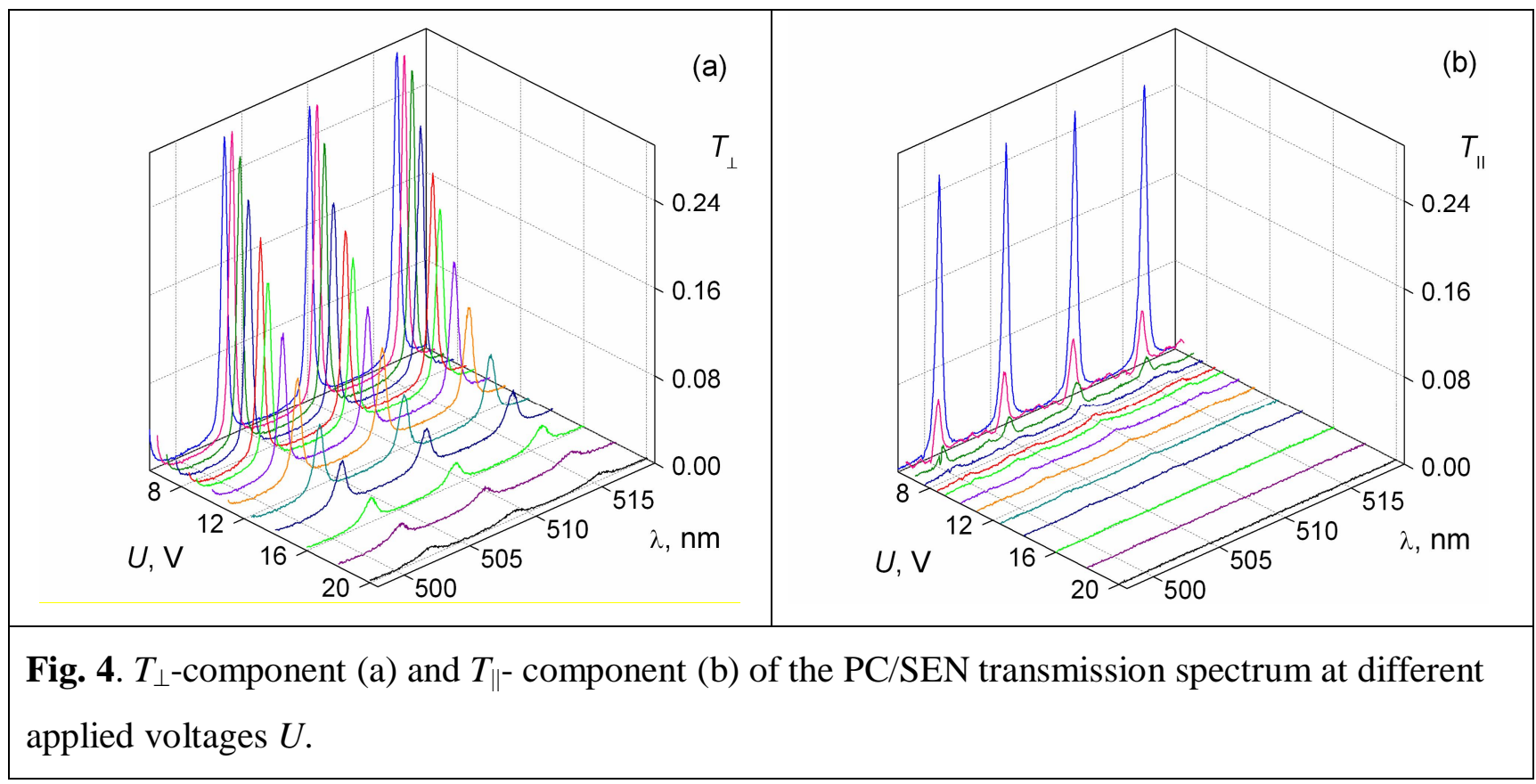

As an example, Fig. 5 shows field dependences of the peaks of neighboring defect modes at wavelengths of $510(\perp$-mode) and $512 \mathrm{~nm}(\|$-mode). It can be seen that the amplitude of $\|$-mode damps almost to the background PBG level in the voltage range 6.2-7.7 V, which involves a sequence of the convective transitions WD $\rightarrow$ OR $\rightarrow$ GP. First, the $e$-wave is scattered on WD and OR phase gratings [20]; therefore, the mode amplitude decreases due to spatial redistribution of the wave energy in the direction perpendicular to the initial propagation direction. The spatial Fourier analysis of the convective transition WD $\rightarrow$ GP for MBBA shows that the grid pattern (Fig. 2d) is the complex 3D flow of the nematic [21]. It is important that the diffraction efficiency of the domain grating in the zero diffraction maximum for the radiation whose polarization coincides with the LC orientation direction is close to unity [22]. Thus, the rapid damping of the parallel modes in the very narrow voltage range originates mainly from the diffraction loss. Then, in the DSM regime, the medium becomes strongly nonuniform for the $e$-wave. In virtue of the large birefringence of the nematic, fluctuations of refractive index $n_{\text {eff }}$ sharply grow and, after passing through such a medium, the direction of the $e$-wave becomes random even in a thin layer [23]. On the contrary, the behavior of transverse modes is relatively stable against convection processes (Fig. 5). For example, 
at a voltage of $7.7 \mathrm{~V}$, the amplitude of $\perp$-mode $(\lambda=510 \mathrm{~nm})$ decreases by a factor of only 1.2 , which can be caused by distortion of the initial direction of propagation of the $o$-wave, which occurs at the strongly nonuniform director orientationa $\mathbf{n}$ in the LC volume, although it is weaker as compared with the $e$-wave [23].

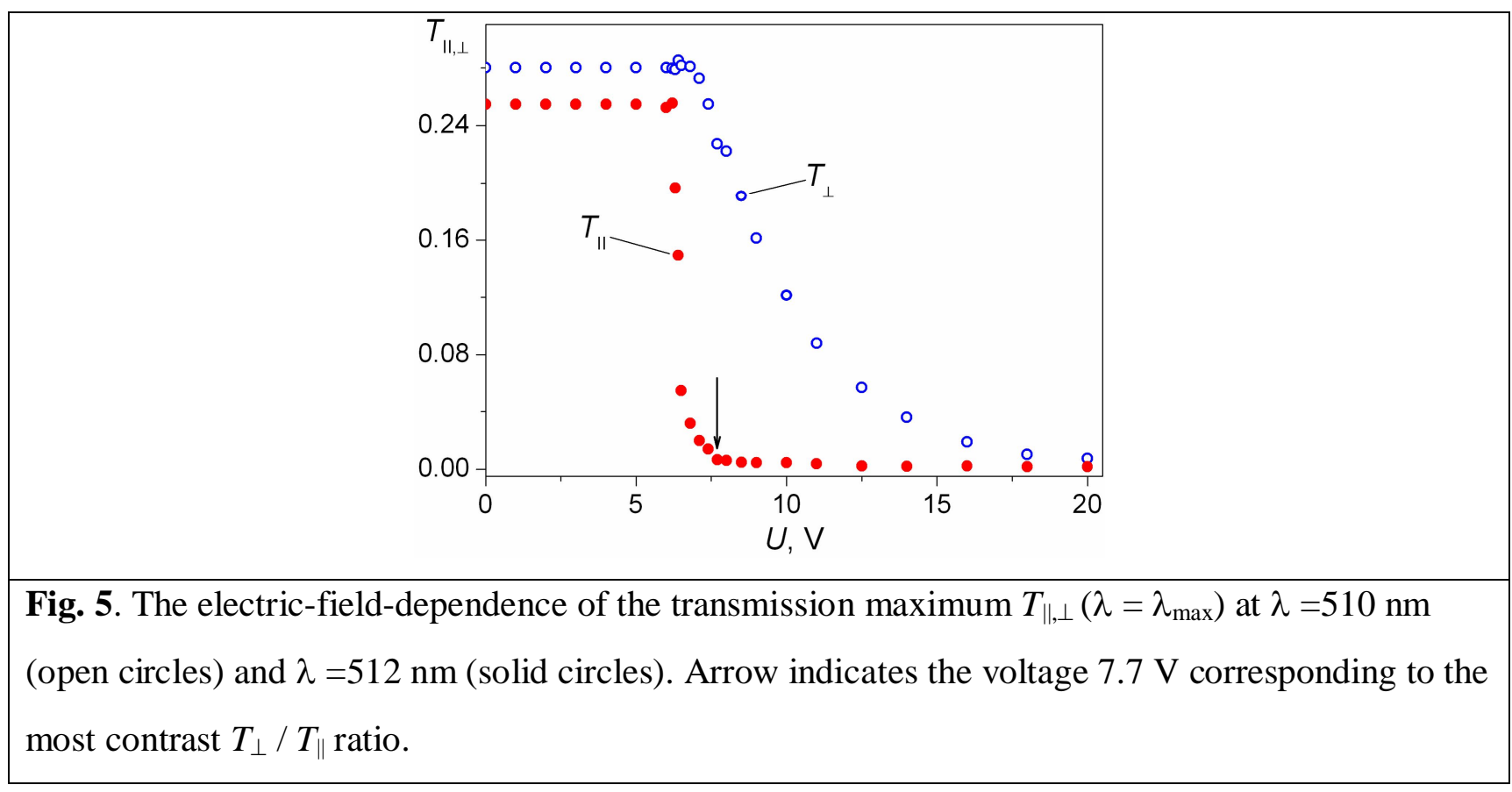

As one can see from results presented in Fig. 5 the choice of a voltage of $7.7 \mathrm{~V}$ is caused by the contrast at the polarization switching of the defect mode from the transparent to optically closed state, which is almost optimal. At the deviation of the electric vector of the light wave from the direction parallel or perpendicular to the rubbing direction $\mathbf{n}$, the modes of the both types are simultaneously excited in the photonic structure. The energy distribution between them depends on the value of this deviation. At the chosen voltage, transmission of the samples at the wavelengths of $\|$-modes is almost completely blocked by the diffraction loss. Only undamped $\perp$-modes occur in the spectrum. Their intensity is a continuous function of the angle between the rubbing direction and plane of polarization of the light normally incident onto the sample, and the fixed spectral position is caused by the stable spatial distribution of the refractive index of the medium. Figure 6 illustrates the controlled transmission of defect modes in the PC/SEN structure at the PBG center upon variation of the light polarization direction. The experimental spectra were obtained at fixed positions of the polarizing element, which are changed with a pitch of $5^{\circ}$. The voltage applied to the sample $(7.7 \mathrm{~V})$ remains invariable. As an example, Figure 7 shows the defect mode attenuation curve $T(\alpha)$ at the wavelength $\lambda=510 \mathrm{~nm}$. 


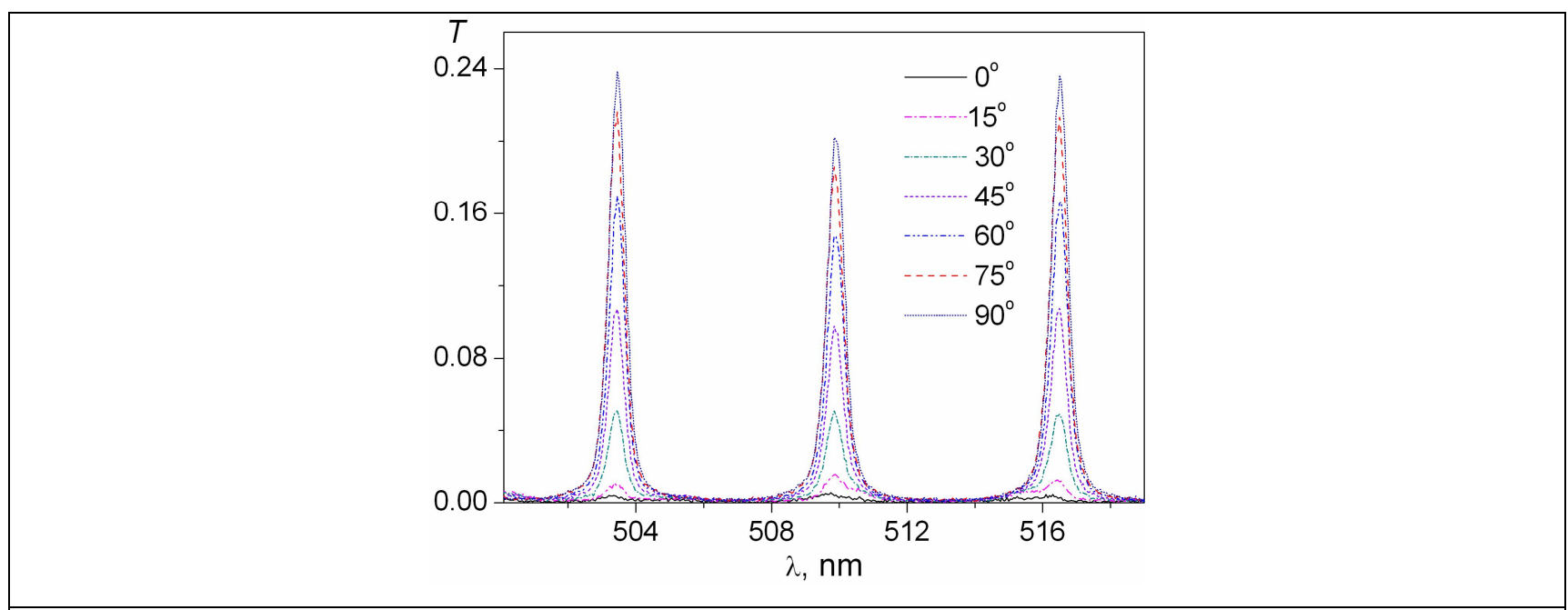

Fig. 6. Transmission spectra of the PC/SEN cell for the various angles $\alpha$ between the rubbing direction $\mathbf{n}$ and light polarization. Operating ac voltage is $7.7 \mathrm{~V}$.

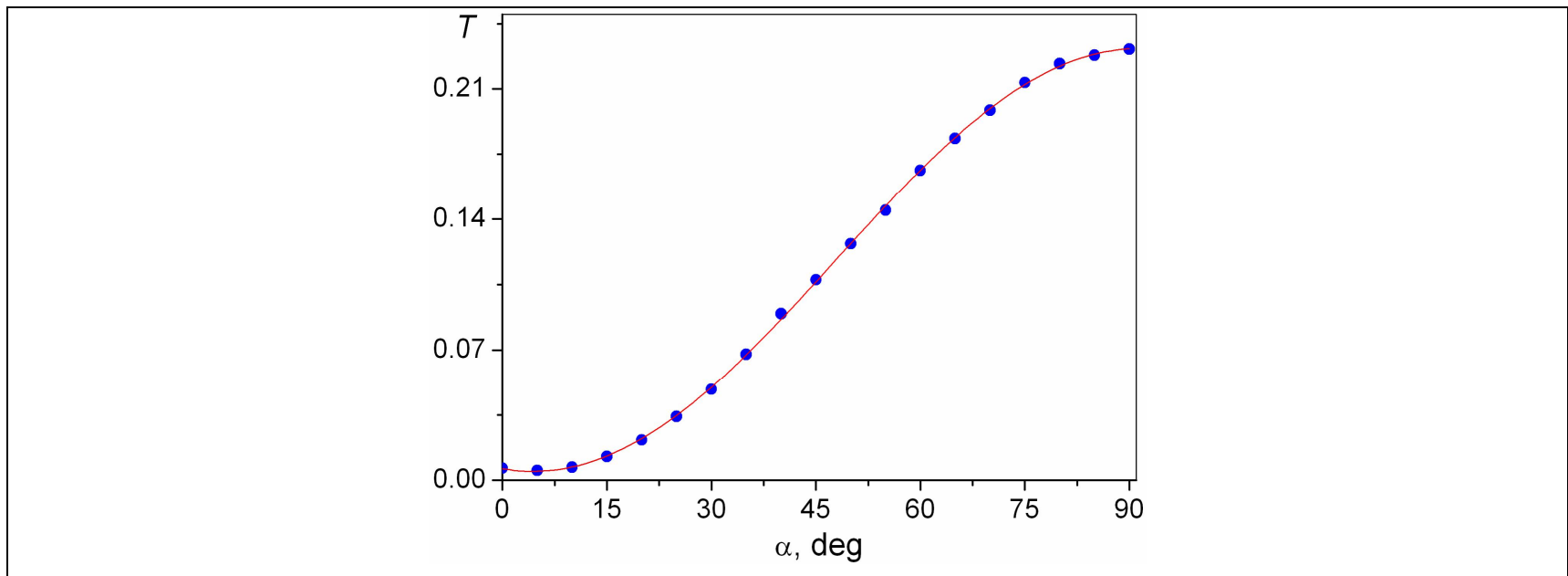

Fig. 7. Defect mode attenuation in photonic crystal cell at wavelength $510 \mathrm{~nm}$ as function of $\alpha$. Experimental data are indicated by circles; solid line is an interpolation.

\section{Conclusion}

Rotation of the plane of polarization of the light incident onto the photonic structure due to rotation of the polarizing element with frequency $v$ leads to the modulation of the intensity of defect modes in the spectrum and, consequently, to the modulation of sample transmittance $T(v)$. The modulation frequency $v$ is limited only by the type of polarization rotators used. The estimated modulation depth $k=\left(T_{\max }-T_{\min }\right) /\left(T_{\max }+T_{\min }\right) \sim 95 \%$. Obviously, coefficient $k$ will significantly increase with increasing quality factor of the microcavity, which, in turn, depends on the number of dielectric layers deposited onto the PC mirrors. 
The proposed technique for modulating modes in the photonic structure involving a nematic with the spatially extended director field as an active control element can be used for filtering, switching, and optical modulation of light in photonics and optoelectronics applications.

\section{Acknowledgements}

The work was supported by the National Academy of Sciences of Belarus and the Russian Academy of Sciences in the frame of the InterAcademy (NASB - SB RAS) Integration Project, and Taiwan - Russian Common Project, and the Belarusian Republican Foundation for Fundamental Research (project F15SB-039).

\section{References}

[1] Joannopoulos JD, Johnson SG, Winn JN, Meade RD. Photonic Crystals: Molding the Flow of Light. Princeton: Princeton University Press; 1995.

[2] Busch K, Lölkes S, Wehrspohn RB, Föll H. Photonic Crystals: Advances in Design, Fabrication, and Characterization. Berlin: John Wiley \& Sons; 2004.

[3] Busch K, von Freymann G, Linden S, Mingaleev SF, Tkeshelashvili L, Wegener M. Periodic structures for photonics. Phys Rep 2007;444:101-202.

[4] Shabanov VF, Vetrov SYa, Shabanov AV. Optics of Real Photonic Crystals: Liquid Crystal Defects, Irregularities. Novosibirsk: SB RAS Publisher; 2005 (in Russian).

[5] Kitzerow H. Tunable photonic crystals. Liq Cryst Today 2002;11:3-7.

[6] Ozaki R, Matsui T, Ozaki M, Yoshino K. Electro-tunable defect mode in one-dimensional periodic structure containing nematic liquid crystal as a defect layer. Jpn J Appl Phys 2002;41:L1482-4.

[7] Arkhipkin VG, Gunyakov VA, Myslivets SA, Gerasimov VP, Zyryanov VYa, Vetrov SYa, Shabanov VF. One-dimensional photonic crystal with a planarly oriented nematic layer: temperature and angular dependence of the spectra of defect modes. JETP 2008;106:388-98.

[8] Gunyakov VA, Myslivets SA, Parshin AM, Zyryanov VYa, Arkhipkin VG, Shabanov VF. Magnetic-field control of the transmission of a photonic crystal with a liquid-crystal defect. Tech Phys 2010;55:1484-9.

[9] Zyryanov VYa, Myslivets SA, Gunyakov VA, Parshin AM, Arkhipkin VG, Shabanov VF, Lee W. Magnetic-field tunable defect modes in a photonic-crystal/liquid-crystal cell. Opt Express 2010;18:1283-8.

[10] Arkhipkin VG, Gunyakov VA, Myslivets SA, Zyryanov VYa, Shabanov VF, Lee W. Electroand magneto-optical switching of defect modes in one-dimensional photonic crystals. JETP 2011;112:577-87. 
[11] Hsiao YC, Wu CY, Chen CH, Zyryanov VYa, Lee W. Electro-optical device based on photonic structure with a dual-frequency cholesteric liquid crystal. Opt Lett 2011;36:2632-4.

[12] Bulgakov EN, Sadreev AF, Gerasimov VP, Zyryanov VYa. Resonant angular conversion in a Fabry-Perot resonator holding a dielectric cylinder. J Opt Soc Am A 2014;31:264-7.

[13] Buka A, Kramer L. Pattern Formation in Liquid Crystals. New York: Springer-Verlag; 1996.

[14] Loiko VA, Konkolovich AV, Miskevich AA. Optical Model of Transient Light Scattering in Ferroelectric Liquid Crystals. JETP 2009;108: 535-45.

[15] Loiko VA, Konkolovich AV, Miskevich AA. Transient light scattering in helix ferroelectric liquid crystal cells. Liq Cryst 2009; 36: 365-370.

[16] Williams R. Domains in liquid crystals. J Chem Phys 1963;39:384-8.

[17] Ribotta R, Joets A. Oblique roll instability in an electroconvective anisotropic fluid. Phys Rev Lett 1986;56:1595-7.

[18] Joets A, Ribotta R. Localized bifurcations and defect instabilities in the convection of a nematic liquid crystal. J Stat Phys 1991;64:981-1005.

[19] John T, Behn U, Stannarius R. Fundamental scaling lows of on-off intermittency in a stochastically driven dissipative pattern-forming system. Phys Rev E 2002;65:046229-13.

[20] Carroll TO. Liquid-crystal diffraction grating. J Appl Phys 1972;43:767-70.

[21] Yamazaki H, Kai S, Hirakawa K. Spatial Fourier analysis of convective cells in the region from the Williams domain to the grid pattern. J Phys Soc Jpn 1983;52:1878-81.

[22] Blinov LM, Yablonsky SV. Electrohydrodynamic instability as a nonequilibrium phase transition. Sov Phys Crystallogr 1982;27:936-40.

[23] Baranova NB, Zel'dovich BYa. Enhanced transmission of an unoriented mesophase of nematics for the ordinary wave. JETP Lett. 1980;32:622-5. 